\title{
Razonamiento estratégico que promueve el comportamiento persistente: un enfoque experimental
}

\author{
Strategic Reasoning that promotes persistent \\ behavior: an experimental approach
}

Federico Torres-Carballo'

Yarima Sandoval-Sánchez $z^{2}$

Torres-Carballo F; Sandoval-Sánchez, Y.

Razonamiento estratégico que promueve el comportamiento persistente: un enfoque experimental. Tecnología en Marcha.VI Encuentro de Investigación y Extensión. Pág

2 Escuela de Computación, Instituto de Tecnología de Costa Rica, Cartago, Costa Rica. Correo electrónico: ysandoval@itcr.ac.cr. 


\section{Palabras claves}

Comportamiento estratégico; las diferencias culturales; economía experimental; juegos de adivinanzas.

\section{Resumen}

La presente investigación tiene como objetivo aplicar técnicas experimentales para estudiar el comportamiento estratégico utilizado en los juegos de "esconder y buscar" (Hide \& Seek en inglés) como un marco para representar situaciones empresariales más reales y elaboradas. Para promover el pensamiento estratégico y la persistencia, se aplican reiterados juegos coincidentes entre desconocidos, y uno de ellos con una dotación fija (pago inicial que disminuye en cada ronda) con la posibilidad de abandono en cualquier ronda y manteniendo el resto de la dotación inicial. De hecho, el razonamiento estratégico se encuentra en el desvío de las elecciones al azar a lo largo de las repeticiones y en la tasa de abandono del juego, la aversión a la pérdida y las diferencias culturales que parecen enmarcar diversos comportamientos estratégicos.

\section{Keywords}

Strategic behavior; cultural differences; economy experimental; guessing games.

\begin{abstract}
This research aims to apply experimental techniques used to study the strategic behavior in games of Hide and Seek, as a framework for representing business situations more realistic and elaborate. To promote strategic thinking and persistence, repeated matching games between strangers are applied, and one with fixed an allocation (down payment decreases each round) with the possibility of abandonment in any round and keeping the rest of the initial allocation. In fact, the strategic reasoning is the diversion of random choices along of repetition and dropout rates of gambling, loss aversion and cultural differences that seem to frame various strategic behaviors.
\end{abstract}

\section{Introducción}

Los juegos del tipo "esconder y buscar" son utilizados para modelar o predecir el comportamiento de las personas en las interacciones estratégicas. Estos juegos pueden representar, convincentemente, problemas que son el centro de muchos entornos económicos, políticos y sociales; o bien, problemas en aplicaciones militares y de seguridad (Crawford e Iriberri, 2007). Desde los primeros estudios en 1993, los resultados de estos juegos mostraron un comportamiento que difiere de lo esperado teóricamente ¿Podría determinado comportamiento de razonamiento estratégico ser modelado en la estructura de un simple juego? ¿Podrían las recompensas económicas promover este tipo de esfuerzo persistente? Estas son preguntas que tienen varias implicaciones para los negocios y el razonamiento estratégico en puestos ejecutivos.
La presente investigación, tiene como objetivo aplicar las técnicas experimentales para el estudio de estos desafíos. Resguardamos el texto y la estructura de estudios previos al experimentar con dos tratamientos: un tratamiento basado en el de Rubinstein, Tversky y Heller (1996) y un tratamiento con una dotación económica inicial y la posibilidad de abandono. Para evitar sesgos, hemos alternado el orden del tratamiento en las sesiones. Las sesiones se desarrollaron en España y Costa Rica para el debido análisis transcultural.

Para promover el pensamiento estratégico y la persistencia y, siguiendo a Eliaz y Rubinstein (2009), se aplicaron reiterados juegos coincidentes entre desconocidos. Uno de ellos fue con una dotación fija (pago inicial, que disminuye en cada ronda) con la posibilidad de abandono en cualquier ronda, y conservando el sujeto el resto de la dotación. 


\section{Juegos del tipo "esconder y buscar"}

Hay dos grupos de juegos "esconder y buscar": juegos de colaboración y los juegos competitivos. En los juegos de colaboración, como el juego de mensajes (también llamado juego de coordinación), el jugador 2 tiene que encontrar el mensaje que el jugador I esconde en una de varias cajas. El jugador I y el jugador 2 ganan si ambos seleccionan la misma opción. Los juegos competitivos son el juego del tesoro y el juego de minas, en el primero, el jugador I (el que oculta) coloca un tesoro en una de varias cajas y el jugador 2 (el que busca) tiene que encontrar el tesoro. En el juego de minas, el buscador tiene que evitar la mina que el ocultador colocó en una de las cajas.

La ecuación (I) representa el juego "esconder y buscar" (G), tal cual Rubinstein y Tversky enunciaron en su primer trabajo (1993), Ai es el conjunto de alternativas (opciones) disponibles para el jugador $i$, y Ui es la función de recompensa del jugador i. La ecuación (2) y (3) expresan la recompensa para el jugador I y 2 , respectivamente.

$$
\begin{array}{r}
G=(A 1, A 2, U 1, U 2) \\
U I(a 1, a 2)=1 \text { if } a 1 \neq a 2 \text { y } 0 \text { por otra parte } \\
U 2(a 1, a 2)=I \text { if } a 1=a 2 \text { y } 0 \text { por otra parte }
\end{array}
$$

El juego presenta un equilibrio de Nash con estrategias mixtas. Cada jugador podría seleccionar cualquier elemento, por lo que cada una de cuatro opciones tiene una probabilidad de 0,25. La prueba de este asunto son las consideraciones estratégicas a las que induce el juego. En equilibrio, el jugador I elegirá la opción que cree que es la menos probable para el jugador 2. De manera similar y equilibradamente, el jugador 2 elegirá la opción que cree es la más probable del jugador 1 .

Por lo tanto, la probabilidad de equilibrio en cualquier acción para ambos jugadores, no puede exceder de 0,25; y finalmente, ambos jugadores establecen iguales probabilidades para las cuatro opciones, por lo que el jugador 2 , el buscador, tiene 0,25 de oportunidad de ganar el juego y el jugador I, el ocultador, tiene 0,75.

Rubinstein, Tversky y Heller realizaron muchas variaciones al experimentar con esta versión del juego "esconder y buscar" en 1993 y 1996, todos ellos se centraron en mostrar la existencia de una Estrategia
Naiive que se produce por el efecto de tener una marca destacada en el conjunto de decisiones. Ellos probaron con distintos grupos de etiquetas (imágenes, símbolos o palabras positivas, negativas o neutrales) y todos los resultados fueron similares.

Explicaron los juegos a los estudiantes como "historias", probablemente incrementando la comprensión por parte de los sujetos. Por ejemplo, Rubinstein dijo a los buscadores: "Usted y otro estudiante jugarán el siguiente juego: su oponente ha escondido un premio en una de cuatro cajas alineadas. Las cajas están marcadas de la siguiente manera: $A, B, A, A$. La meta de ustedes es, por supuesto, encontrar el premio. El objetivo de su oponente es que ustedes no lo encuentren. A ustedes les es permitido abrir solamente una caja. ¿Cuál caja van a abrir?". A los ocultadores les fue expuesta una historia semejante. De esta forma la estructura, incluyendo el orden y etiquetado de las ubicaciones, fue públicamente anunciada.

Esta historia le resta neutralidad al conjunto de locaciones de dos maneras: se explicó que la ubicación "B" se distingue por su etiqueta, por lo que, según Thomas Schelling (1960), se convierte en un punto sobresaliente. Y las dos ubicaciones "A" del final, a pesar de no ser distinguidas por su etiqueta, pueden ser inherentemente destacadas (ver Rubinstein, Tversky and Heller 1996). Rubinstein y Tversky vieron que esas dos distinciones interactuaron, y le dieron a la ubicación restante, la "A" central, su propia marca de singularidad como "la ubicación menos destacada".

Ellos confirmaron el efecto tan visible mediante la ejecución de dos juegos colaborativos. Sus estudios muestran que en los juegos de Coordinación, la distribución de elecciones fue notablemente diferente a la esperada por la casualidad porque más del $50 \%$ de los jugadores eligieron el punto focal en todos los juegos (sin importar cuál conjunto de etiquetas fue usado). Este hecho confirmó el efecto del punto sobresaliente $y$, en contraste, en el juego de descoordinación, la distribución de elecciones del punto focal fue muy similar a la esperada por la selección al azar.

Finalmente, ellos llegaron a la conclusión de que los datos indican que, con la presencia de un solo ítem distintivo, los jugadores son capaces de alcanzar un nivel de coordinación razonable (alrededor de 65\%); y que, en ausencia de un método efectivo para alcanzar la descoordinación, los jugadores escogieron más o menos al azar. 
Además, ellos mostraron que en los juegos Competitivos (juegos de Tesoros y Minas), los resultados para los buscadores y los ocultadores fueron contrarios a los que predice el análisis teórico del juego. Ellos descubrieron que ambos jugadores, ocultadores y buscadores, tienden a evadir los elementos del final. Rubinstein y colaboradores señalaron que esta tendencia fue observada a la hora de elegir productos de un estante en el supermercado, seleccionar la caseta de un baño público, - eligiendo un símbolo arbitrario, de acuerdo a Christenfeld (1995).

Crawford and Iriberri (2007) propusieron una explicación para el comportamiento de los juegos competitivos con un modelo de estrategia de Nivel $\mathrm{K}$ de razonamiento. Ellos respaldaron su investigación basados en los experimentos de Rubenstein y coautores (1993, 1996 y 1999) y compararon explicaciones alternativas de las desviaciones sistemáticas del equilibrio. Ellos usaron un enfoque econométrico para mostrar que el Nivel $\mathrm{K}$ de razonamiento provee una explicación aceptable a la desviación en los datos de Rubinstein y coautores.

El cuadro I muestra un resumen de los juegos explorados en los últimos veinte años. Los diferentes investigadores han estado trabajando con la teoría de los juegos de "esconder y buscar", haciendo variaciones del experimento original ejecutado por Rubenstein y Tversky en 1993.

El cuadro I muestra el tipo de juegos usados para el experimento. Como mencionamos anteriormente, el experimento original funcionaba con dos conjuntos de etiquetas: letras ("A", "A", "B", "A") y números $(I, 2,3,4)$ y cuatro opciones; sin embargo, otros autores implementaron experimentos con un mayor número de conjuntos de etiquetas y/o un número de opciones diferente. Por ejemplo, Rojo y coautores trabajaron con 18 conjuntos de etiquetas, que fueron clasificadas como etiquetas positivas ( 6 conjuntos), negativas ( 6 conjuntos) y neutrales ( 6 conjuntos), y una variación del juego con 8 opciones o cajas (ver Rojo, Sugden y Hargreaves-Heap 20 I I).

Además, el cuadro I muestra la categoría y el tipo de juego implementado en cada experimento, es importante entender la diferencia entre los juegos competitivos y los colaborativos: en los juegos competitivos son juegos de suma cero, lo que significa que cuando el jugador 2 gana, el jugador I pierde y viceversa, por el contrario, en los juegos colaborativos ambos jugadores son ganadores si ellos escogen la misma opción (en el tipo coordinado) o si escogen diferente opción (en el tipo descoordinado).

Todas estas variaciones son estructuradas como un juego de una sola ronda, y todas ellas usaron una etiqueta sobresaliente, lo que hace que el juego no sea neutral por las ubicaciones establecidas.

\section{Teoría de razonamiento de Nivel K}

Como mencionamos anteriormente, Crawford and Iriberri (2007) exploraron la especificación de los modelos de razonamiento de Nivel K para los juegos ejecutados en escenarios no neutrales; además, trataron de establecer una relación entre los resultados de Rubinstein, Tversky y Heller (1996),

Cuadro I. Resumen de artículos de los juegos de adivinanzas

\begin{tabular}{|l|c|l|}
\hline \multirow{2}{*}{ Autores } & \multicolumn{2}{|c|}{ Tipo de juego } \\
\cline { 2 - 3 } & Competitivo & \multicolumn{1}{c|}{ Colaborativo } \\
\hline $\begin{array}{l}\text { Rubinstein y } \\
\text { Tversky (1993) }\end{array}$ & $\begin{array}{c}\text { Tesoro } \\
\text { Mina }\end{array}$ & Mensaje (Coordinación) \\
\hline Rubinstein, Tversky y Heller (1996) & $\begin{array}{l}\text { Tesoro } \\
\text { Mina }\end{array}$ & $\begin{array}{l}\text { Coordinación } \\
\text { Descoordinación }\end{array}$ \\
\hline $\begin{array}{l}\text { Crawford \& Iriberri } \\
(2007)\end{array}$ & Tesoro & Mensaje (Coordinación) \\
\hline Rojo et al. (2011) & $\begin{array}{l}\text { Tesoro } \\
\text { Mina }\end{array}$ & Mensaje (Coordinación) \\
\hline Heinrich y Wolff (2012) & Tesoro & --- \\
\hline
\end{tabular}


y el trabajo experimental sobre el pensamiento estratégico y trajeron evidencia para dar soporte a las respuestas iniciales de los juegos.

Ellos basaron su discusión en los resultados de Rubinstein, Tversky and Heller (1996) y resultados relacionados, utilizando un modelo de respuestas iniciales no equilibrado para los juegos basados en la teoría del razonamiento de "Nivel K". Esta teoría fue construida sobre el análisis de Bacharach y Stahl (1997), acerca de una versión simplificada de los juegos de Rubinstein, Tversky and Heller.

Los modelos de Nivel $K^{\prime}$ tienen un fuerte respaldo experimental, lo que debería aliviar la preocupación de que, una vez que uno afloja el equilibrio, cualquier cosa es posible.

El modelo de Nivel $K$ es una teoría que distingue a las personas de acuerdo con su nivel de razonamiento estratégico. Por ejemplo, el Nivel 0 (N0) son personas que no emplean un razonamiento estratégico, ellos simplemente eligen una opción. Nivel I (NI) es el primer nivel en emplear un pensamiento estratégico, las personas eligen la mejor respuesta, a la acción de las personas del Nivel 0; de igual forma, las personas del Nivel 2 (N2) buscan un razonamiento estratégico más alto, asumiendo que su oponente ha usado una estrategia de NI, y así sucesivamente. Es importante señalar que esta teoría no hace ninguna presunción acerca de lo que las personas de No eligen, o del por qué y cómo está distribuida la población en los diferentes niveles de razonamiento.

Además, Crawford e Iriberri enfocaron su trabajo en el juego de "esconder y buscar", y juegos relacionados ejecutados en escenarios que no son neutrales a la hora de elaborar los conjuntos de opciones pero neutrales en lo que se refiere a las recompensas.

Aunque el aprendizaje puede converger al equilibrio sobre el tiempo, ellos siguieron a Rubinstein, Tversky y Heller (1996) al estudiar las respuestas iniciales de los jugadores. Ellos evidenciaron que el comportamiento observado en los juegos de "esconder y

I Los modelos de nivel K fueron presentados por Stahl y Paul Wilson (1994, 1995) y Rosemarie Nagel (1995) y ampliados por Teck- Hua Ho et al. (1998), Costa-Gomes et al. (200I), Vincent Crawford (2003), Colin Camerer et al. (2004), Costa-Gomes and Crawford (2006), and Crawford and Nagore Iriberri (2005). buscar", puede ser explicado por el comportamiento estratégico. De hecho, su análisis sugiere niveles altos de sofisticación del jugador, tratándose de niveles de razonamiento.

Rojo, Sugden y Hargreaves-Heap (20II) propusieron una prueba diferente de la teoría de Nivel K. Ellos trabajaron con tres tipos de juegos: coordinación (mensaje), descoordinación y tesoro y utilizaron los cuatro conjuntos de etiquetas de Rubinstein, Tversky y Heller (1996), además de un conjunto adicional de 8 etiquetas.

Al final de su experimento, ellos resumen sus conclusiones en tres comportamientos: I. Los individuos eligen opciones sobresalientes en los juegos de Coordinación Pura, pero no con probabilidad I, sin embargo, la probabilidad es relativamente alta con el número de posibles opciones. 2. Los individuos eligen al azar en los juegos de descoordinación. 3. Los ocultadores tratan de elegir al azar, pero eligen con menor frecuencia la opción sobresaliente, mientras que los buscadores tratan de elegir al azar pero escogen con mayor frecuencia la respuesta sobresaliente.

\section{Nuestro experimento}

Las sesiones fueron ejecutadas en el Laboratorio de Economía Experimental (LEXTEC), en el Instituto Tecnológico de Costa Rica, y en el Laboratorio para la Investigación en la Economía Experimental (LINEEX), en la Universidad de Valencia. Los individuos fueron elegidos de un grupo de estudiantes en cada universidad. Un nivelado número de individuos (22 en Costa Rica, 24 en España) participaron en cada sesión. El cuadro 2, muestra el total de participantes por país. La computadora asoció al azar a los individuos en parejas anónimas (i.e., utilizamos un diseño de "desconocidos"). Cada pareja jugó 10 juegos competitivos de "esconder y buscar" repetidamente en cada uno de los dos tratamientos. Para el proceso de diseño e implementación de los experimentos, usamos el software Z-Tree (Herramienta de software creada para experimentos económicos en la Universidad de Zurich).

El software Z-Tree es una herramienta especializada para el desarrollo e implementación de experimentos económicos, permitiendo juegos dinámicos, mercados, negociación, subasta y otros. Al final de cada sesión las recompensas fueron pagadas en efectivo a cada jugador: la cantidad promedio 
Cuadro 2. Número de participantes en los experimentos

\begin{tabular}{|l|c|c|}
\hline & Cartago, CR & Valencia, España \\
\hline $\begin{array}{l}\text { TI, } \\
\text { T2* }\end{array}$ & 44 & 48 \\
\hline $\begin{array}{l}\text { T2, } \\
\text { TI** }\end{array}$ & 44 & 48 \\
\hline
\end{tabular}

*Se aplicó primero en tratamiento I, luego el tratamiento 2

**Se aplicó primero el tratamiento 2, luego el tratamiento I

pagada a los individuos en Costa Rica fue de $\$ 4$ y en España fue de $\$ 6,5$. El proceso completo tomó alrededor de una hora.

\section{Tratamiento I}

Cada participante fue asignado a desempeñar cualquiera de los dos papeles, el de ocultador o el de buscador, a través de las 10 rondas. Los jugadores fueron informados de que serían emparejados al azar con una persona que jugaría el papel opuesto al suyo. Se les dijo que la tareas de los ocultadores es "esconder el tesoro detrás de uno de los cuatro ítems", conservamos la redacción y el diseño de estudios previos (ver figural). Mientras que el papel del buscador es "adivinar la ubicación del tesoro", si el buscador adivina correctamente, gana $25 \mathrm{ECUs}^{2}$ y el ocultador no recibe nada; si el buscador no adivina correctamente, entonces, no recibe nada y el ocultador recibe 25 ECUs en esta ronda.

\section{Tratamiento 2}

Diseñamos este segundo tratamiento como una variante del primero. Como los individuos del tratamiento I, los del tratamiento 2 tuvieron que completar un conjunto de 10 decisiones con respuestas entre los mismos 4 ítems (véase figura I), esconder o buscar un "tesoro" para ganar 25 ECUs. Pero, en este caso, en cada ronda los buscadores deben elegir entre continuar o abandonar porque al inicio del tratamiento recibieron un monto de 50 ECUs y este monto decrecía 5 ECUs por cada ronda jugada.

\section{Resultados}

\section{Primera ronda}

Primeramente, describimos los resultados de la primera ronda porque podemos compararlos con los resultados de los experimentos previos. El cuadro 3 muestra que contrario al análisis teórico del juego y como ha sucedido en estudios anteriores, los jugadores no eligieron los 4 ítems con la misma probabilidad. La distribución de frecuencia relativa presenta que en Costa Rica los dos jugadores, en cualquier orden de aplicación del tratamiento, la "opción menos sobresaliente", la tercera "A" se encuentra a la cabeza con más del $40 \%$ de elecciones. La prueba $\chi^{2}$ indica que los buscadores se

\title{
Estrategias naive in juegos de suma zero
}

\author{
Rbiestein y Tversky 1993
}

Típicas instrucciones para el buscador (similar para el ocultador):

\begin{abstract}
Su oponente ha escondido un premio en una de las cuatro cajas alineadas. Las cajas están marcadas como se muestra a continuación: A, B, A, A. Su objetivo es, por supuesto, encontrar el premio. El objetivo de su oponente es que usted no lo encuentre. Se le permite abrir solo una caja.
\end{abstract}

¿Cuál caja va a abrir?

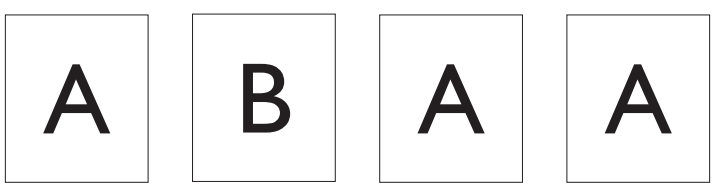

Figura I. Figura tomada de la redacción original.

2 ECU=Experimental economic unit 
apartan significativamente de la elección al azar ,y los ocultadores lo hacen en la aplicación del tratamiento I-tratamiento2 (TI-T2).

En el caso de España, esta tendencia a la opción menos sobresaliente no es tan extrema. En el cuadro 4, la distribución de la frecuencia relativa muestra que la tercera "A" predomina en el buscador en ambas secuencias, y para el ocultador en la secuencia TI-T2. La prueba $x^{2}$ es relevante solo para el buscador en el orden T2-TI.

\section{Todas las rondas}

El cuadro 5 muestra la información de Costa Rica en las diez rondas por orden, tratamiento y tipo de jugador. No hay un aspecto sobresaliente, excepto en el orden T2-TI. Los individuos delT2 eligieron en un 36\% la tercera "A" con una desviación importante de azar en la Prueba $x^{2}$.

La información de España en el cuadro 6 presenta un resultado un tanto diferente. En el orden T2-TI todos los tratamientos para ambos jugadores son relevantes en la prueba $x^{2}$, y la distribución de frecuencia relativa mostró que los ocultadores evadieron las tercera "A" con un 9\% y $12 \%$ de las elecciones, muy lejos del $25 \%$ predicho y que prefieren la primera "A". Por el contrario, los buscadores prefieren en el T2 la tercera " $A$ ", y eligen con mayor frecuencia la primera " $A$ " en el TI. En el orden TI-T2, los ocultadores evaden la tercera "A" en ambos tratamientos, pero únicamente en el tratamiento I se apartan significativamente de la elección al azar.

Las Pruebas $x^{2}$ en los buscadores no son significativas, ellos parecen evadir la tercera "A", también en elT2 en un $20 \%$ de respuestas, pero en el TI, no hay una tendencia clara. Estos resultados tienden a coincidir con Rojo, Suden y Hargreaves-Heap (20 I I).

\section{Abandono}

El cuadro 7 muestra cuántos juegos ejecutaron los buscadores en Costa Rica antes de abandonar. Es de hacer notar que, casi el $60 \%$ de los individuos se quedaron a las 10 rondas, y menos del 15\% renunció luego de la quinta ronda. En contraste el cuadro 8, muestra que solo el $17 \%$ de los individuos en España, en el orden TI-T2, se quedaron hasta la décima ronda, y el 54\% de individuos decidieron no continuar antes de la quinta ronda. En el orden $\mathrm{T} 2-\mathrm{TI}$, en las sesiones que comienzan con abando-

Cuadro 3. Datos de Costa Rica: Análisis de la primera ronda

\begin{tabular}{|c|c|c|c|c|c|}
\hline & A & B & A & A & P* \\
\hline Ocultador & & & & & \\
\hline TI-T2 & $14 \%$ & $9 \%$ & $55 \%$ & $23 \%$ & 0,011 \\
\hline T2-TI & $23 \%$ & $14 \%$ & $45 \%$ & $18 \%$ & 0,153 \\
\hline Buscador & & & & & \\
\hline TI-T2 & $9 \%$ & $18 \%$ & $64 \%$ & $9 \%$ & 0,000 \\
\hline T2-TI & $9 \%$ & $5 \%$ & $77 \%$ & $9 \%$ & 0,000 \\
\hline
\end{tabular}

*Nivel de importancia asociado al resultado de la Prueba $\chi^{2}$

Cuadro 4. España, análisis de la primera ronda

\begin{tabular}{|c|c|c|c|c|c|}
\hline & A & B & A & A & P \\
\hline Ocultador & & & & & \\
\hline TI-T2 & $21 \%$ & $17 \%$ & $38 \%$ & $25 \%$ & 0,506 \\
\hline T2-TI & $46 \%$ & $13 \%$ & $29 \%$ & $13 \%$ & 0,062 \\
\hline Buscador & & & & & \\
\hline TI-T2 & $21 \%$ & $17 \%$ & $42 \%$ & $21 \%$ & 0,300 \\
\hline T2-TI & $8 \%$ & $21 \%$ & $67 \%$ & $4 \%$ & 0,000 \\
\hline
\end{tabular}


Cuadro 5. Costa Rica:Análisis de todas las rondas

\begin{tabular}{|c|c|c|c|c|c|}
\hline \multicolumn{6}{|c|}{ Orden:TI-T2 } \\
\hline & A & B & A & A & $p$ \\
\hline \multicolumn{6}{|c|}{ Ocultador } \\
\hline $\mathrm{TI}$ & $25 \%$ & $23 \%$ & $27 \%$ & $25 \%$ & $0,8 \mid 4$ \\
\hline $\mathrm{T} 2$ & $21 \%$ & $28 \%$ & $20 \%$ & $31 \%$ & 0,137 \\
\hline \multicolumn{6}{|c|}{ Buscador } \\
\hline $\mathrm{TI}$ & $23 \%$ & $28 \%$ & $28 \%$ & $21 \%$ & 0,367 \\
\hline $\mathrm{T} 2$ & $23 \%$ & $28 \%$ & $31 \%$ & $19 \%$ & 0,140 \\
\hline \multicolumn{6}{|c|}{ Orden:T2-TI } \\
\hline & A & B & A & A & $\mathrm{P}$ \\
\hline \multicolumn{6}{|c|}{ Ocultador } \\
\hline $\mathrm{TI}$ & $22 \%$ & $26 \%$ & $24 \%$ & $28 \%$ & 0,550 \\
\hline $\mathrm{T} 2$ & $26 \%$ & $24 \%$ & $28 \%$ & $22 \%$ & 0,642 \\
\hline \multicolumn{6}{|c|}{ Buscador } \\
\hline TI & $26 \%$ & $28 \%$ & $24 \%$ & $22 \%$ & 0,550 \\
\hline $\mathrm{T} 2$ & $14 \%$ & $28 \%$ & $36 \%$ & $22 \%$ & 0,001 \\
\hline
\end{tabular}

Cuadro 6. España, análisis de todas las tablas

\begin{tabular}{|c|c|c|c|c|c|}
\hline \multicolumn{6}{|c|}{ Orden:TI-T2 } \\
\hline & A & B & A & A & $\mathrm{P}$ \\
\hline \multicolumn{6}{|c|}{ Ocultador } \\
\hline $\mathrm{TI}$ & $23 \%$ & $29 \%$ & $19 \%$ & $30 \%$ & 0,045 \\
\hline $\mathrm{T} 2$ & $31 \%$ & $24 \%$ & $20 \%$ & $25 \%$ & 0,513 \\
\hline \multicolumn{6}{|c|}{ Buscador } \\
\hline $\mathrm{TI}$ & $23 \%$ & $29 \%$ & $26 \%$ & $21 \%$ & 0,330 \\
\hline $\mathrm{T} 2$ & $24 \%$ & $32 \%$ & $20 \%$ & $23 \%$ & 0,323 \\
\hline \multicolumn{6}{|c|}{ Orden:T2-TI } \\
\hline & A & B & A & A & P \\
\hline \multicolumn{6}{|c|}{ Ocultador } \\
\hline $\mathrm{TI}$ & $41 \%$ & $32 \%$ & $9 \%$ & $18 \%$ & 0,000 \\
\hline $\mathrm{T} 2$ & $43 \%$ & $26 \%$ & $12 \%$ & $19 \%$ & 0,000 \\
\hline \multicolumn{6}{|c|}{ Buscador } \\
\hline $\mathrm{TI}$ & $33 \%$ & $23 \%$ & $25 \%$ & $20 \%$ & 0,024 \\
\hline $\mathrm{T} 2$ & $21 \%$ & $23 \%$ & $40 \%$ & $17 \%$ & 0,000 \\
\hline
\end{tabular}


Cuadro 7. Costa Rica, juegos ejecutados por el buscador antes de abandonar.

Orden:TI-T2

\begin{tabular}{|c|c|c|}
\hline \# de juegos & Frec. & Porcentaje \\
\hline 10 & 13 & 59 \\
\hline 7 & 1 & 5 \\
\hline 6 & 2 & 9 \\
\hline 5 & 4 & 18 \\
\hline 1 & 1 & 5 \\
\hline 0 & 1 & 5 \\
\hline
\end{tabular}

Orden:T2-TI

\begin{tabular}{|c|c|c|}
\hline \# de juegos & Frec. & Porcentaje \\
\hline 10 & 13 & 59 \\
\hline 9 & 1 & 5 \\
\hline 6 & 1 & 5 \\
\hline 5 & 4 & 18 \\
\hline 3 & 3 & 14 \\
\hline
\end{tabular}

Cuadro 8. España, juegos ejecutados por el buscador antes de abandonar.

\begin{tabular}{|c|c|c|}
\hline $\begin{array}{l}\text { Orden:T1-T2 } \\
\text { \# de juegos }\end{array}$ & Frec. & Porcentaje \\
\hline 1 & 4 & 16,67 \\
\hline 5 & 4 & 16,67 \\
\hline 10 & 4 & 16,67 \\
\hline 2 & 3 & 12,5 \\
\hline 3 & 3 & 12,5 \\
\hline 4 & 2 & 8,33 \\
\hline 6 & 2 & 8,33 \\
\hline 0 & 1 & 4,17 \\
\hline 9 & I & 4,17 \\
\hline $\begin{array}{l}\text { Orden:T2-TI } \\
\text { \# de juegos }\end{array}$ & Frec & Porcentaje \\
\hline 10 & 9 & 37,5 \\
\hline I & 4 & 16,67 \\
\hline 5 & 4 & 16,67 \\
\hline 6 & 2 & 8,33 \\
\hline 7 & 2 & 8,33 \\
\hline 2 & 1 & 4,17 \\
\hline 3 & 1 & 4,17 \\
\hline 4 & 1 & 4,17 \\
\hline
\end{tabular}


no, el $39 \%$ de buscadores permanecieron las diez rondas y solo el 29 \% renunció antes de la quinta ronda. Se observa, además, que los españoles prefieren retirarse en la primera, quinta o décima ronda, en tanto que los costarricenses se retiran mayoritariamente en rondas superiores a la quinta.

\section{Persistencia}

La transición de probabilidades en términos de repetición de la misma respuesta, también fue analizada. Encontramos diferencias substanciales con la predicción del 25\%. La información de España evidencia una cantidad alta de respuestas repetidas: $51 \%$ en los ocultadores y $38 \%$ en los buscadores. Un 16\% de individuos eligieron el mismo ítem durante un tratamiento completo. Por el contrario, la información de Costa Rica revela un 32\% en los ocultadores y un $21 \%$ en los buscadores, con solo un $4 \%$ de individuos eligiendo la misma opción en todo un tratamiento.

\section{Discusión}

Nuestros resultados para el juego de "esconder y buscar" en la primera ronda, fueron consistentes y corroboran la preponderancia de la tercera "A", encontrada en estudios previos.

Las repeticiones del juego permitieron determinar que las elecciones de los individuos difieren persistentemente del comportamiento que se predice al azar. Se observaron otras tendencias como que los ocultadores evaden la tercera "A" o que la primera " $A$ " adquiere relevancia para los buscadores y ocultadores.

Bajo la suposición de razonamiento de Nivel K, tiene mucho sentido que las estrategias aplicadas por los jugadores fueran una persistente desviación del azar. De hecho, existen jugadores que prefieren una solución persistente eligiendo la misma opción en varias rondas sucesivas. Si se analiza desde el marco conceptual que aporta la teoría del modelo de procesamiento dual, se podría considerar que el Sistema I, tan intuitivo, rápido y con una operación asociativa, permite a los individuos realizar una simplificación eficiente de la tarea.

Es plausible que los segundos tratamientos, independientemente del orden, muestran más persistencia que los primeros tratamientos, como si los jugadores estuvieran evadiendo esforzarse luego de muchas rondas. Además, los buscadores, en su estresante papel, evidencian menos persistencia que los ocultadores; y los buscadores costarricenses parecen mostrar un efecto contrario de evitar la repetición con una proporción de respuestas repetidas por debajo de lo predicho.

También, se presentan diferencias muy interesantes en las estrategias aplicadas en cuanto a cuando retirarse. En los sujetos españoles parece comprenderse el juego en tres etapas inicio, medio y fin; en cambio, el sujeto costarricense parece comprenderlo como una tarea a completar al máximo. Se espera seguir este análisis con más información para estudiar estas posibles tendencias o heurísticas estratégicas.

La principal diferencia entre el tratamiento I y el tratamiento 2, es la posibilidad de abandono por parte de los buscadores. Ellos son motivados a abandonar la aversión a la pérdida está presente debido a que los buscadores tienen una cantidad, una dotación inicial que decrece en cada juego. Sin embargo, las pruebas Wilcoxon para muestras relacionadas $(p<0.05)$ indican que no hay diferencias significativas entre el tratamiento I y el tratamiento 2 para todos los grupos en sus respuestas. Caso contrario, las pruebas Kolmogorov-Smirnov $(p<0.05)$ indican que sí hay diferencias significativas en la tendencia al abandono entre los individuos de Costa Rica y España.

Necesitamos más información, pero suponemos dos posibilidades: existe un valor diferente en la aversión a la pérdida y la resiliencia entre los países, y/o existe una estrategia de abandono dirigida por aspectos culturales. En cualquier caso, el comportamiento de abandono no afecta la decisión de cada juego: afecta la estrategia del comportamiento en el proceso de la toma de decisiones.

\section{Investigación futura}

Los resultados del estudio muestran que los buscadores son motivados a abandonar; por lo tanto, la aversión a la pérdida está presente ya que los buscadores poseen una cantidad, una dotación, inicial y pierden una parte de ella en cada ronda del juego.

Para un mejor enfoque de los resultados, es necesario hacer el mismo experimento para recolectar 
más información por tratamiento, por país y repetirlo en otros contextos, con suficiente información siguiendo el trabajo Crawford e Iriberri (2007), para construir un modelo econométrico y, así, dar respuesta a preguntas como:

- ¿Hay un valor distinto de aversión a la pérdida y resiliencia?

- ¿Existe una estrategia de abandono dirigida por aspectos culturales?

La repetición de los juegos de esconder y buscar, como se propone en este documento, puede mostrar distintos comportamientos en procesos de pensamiento estratégico en ambientes cooperativos o competitivos, niveles de aversión a la pérdida o resiliencia de los sujetos en procesos frustrantes de toma de decisiones. Experimentos como estos, calibrados por las diferencias culturales, pueden ser aplicados en seleccionando personal y en el redise- ño de la dinámica de trabajo para evitar resultados indeseados en el proceso de tomar decisiones.

\section{Referencias}

Crawford, V. P., \& Iriberri, N. (2007). Fatal attraction: Salience, Naivete, and Sophistication in Experimental "Hide-and-Seek" games. The American Economic Review, 1731-1750. Eliaz, K., \& Rubinstein, A. (20 I I). Edgar Allan Poe's riddle: Framing effects in repeated matching pennies games. Games and Economic Behavior, 7 I ( I), 88-99.

Rubinstein, A., Tversky, A., \& Heller, D. (1993). Naive Strategies in Zero-sum games. Sackler Institute of Economic Studies.

Rubinstein, A., Tversky, A. y Heller, D. (1996). "Naive Strategies in Competitive Games", in Understanding Strategic Interaction-Essays in Honor of Reinhard Selten, ed. Wulf Albers, Werner Güth, Peter Hammerstein, Bemmy Moldovanu, and Eric van Damme. 394-402. Berlin: Springer-Verlag.

Rubinstein, A. (1999). Experience from a course in game Theory: Pre-and Postclass Problem Sets as a Didactic Device. Games and Economic Behavior, 28 (I), I55-170. 